Supporting Information for

\title{
Trimetallic Mo-/Ni-/Fe-Based Hybrids Anchored on Hierarchical N-CNTs Arrays with Abundant Defects and Interfaces for Alkaline Water Splitting
}

Renjun Zhao, ${ }^{a}$ Xuesong Liu, ${ }^{a}$ Kuan Deng, ${ }^{a}$ Wen Tian, ${ }^{a}$ Kui Ma,${ }^{a}$ Shuai Tan, ${ }^{a}$ Hairong Yue, ${ }^{a}$ Junyi $J i^{a, b, *}$

a School of Chemical Engineering, Sichuan University, Chengdu 610065, P. R. China

b State Key Laboratory of Polymer Materials Engineering, Sichuan University, Chengdu 610065, P. R. China

* Corresponding author.

Junyi Ji, E-mail: junyiji@scu.edu.cn 


\section{SUPPLEMENTARY EXPERIMENTAL SECTION}

Materials characterizations. The morphology and structure evolution were characterized by scanning electron microscopy (SEM, JEOL JSM-7610F), energy dispersive X-ray spectroscopy (EDS), and transmission electron microscopy (TEM, JEOL JEM-F200). The crystallinity was identified by X-ray diffraction (XRD, DX2700, Dandong Haoyuan). X-ray photoelectron spectroscopy (XPS, PHI5000 Versa spectrometer) was conducted to study the elemental valence states.

Electrochemical measurements: All electrochemical measurements were carried out with a Metrohm Autolab M204 electrochemical workstation. The linear sweep voltammetry (LSV) curves were evaluated at $5 \mathrm{mV} \mathrm{s}^{-1}$ in a three-electrode system with $1 \mathrm{M} \mathrm{KOH}$ as electrolyte and corrected by $i R$. The calibration formula is as follows:

$$
\mathrm{E}=\mathrm{E}_{\mathrm{RHE}}-\mathrm{I}^{*} \mathrm{R}_{\mathrm{s}}
$$

$\mathrm{R}_{\mathrm{S}}$ refers to the solution electric resistance. $\mathrm{E}_{\mathrm{RHE}}$ refers to the potential calibrated by the reversible hydrogen electrode (RHE) scale. All the potentials were calibrated to RHE using the equation:

$$
\mathrm{E}_{\mathrm{RHE}}=\mathrm{E}_{\mathrm{Hg} / \mathrm{HgO}}+0.059 * \mathrm{pH}+0.098
$$

The composite electrodes, $\mathrm{Hg} / \mathrm{HgO}$ electrode and carbon rod were used as the working, reference and counter electrodes, respectively. Electrochemical double-layer capacitance $\left(\mathrm{C}_{\mathrm{dl}}\right)$ measurements were carried out by using $\mathrm{CV}$ curves swept at voltage interval of $0.1 \mathrm{~V}$ without Faradaic region and scan rates set as 10, 20, 30, 40, 50 and $100 \mathrm{mV} \mathrm{s}^{-1}$. By plotting the difference between the maximum and minimum values of current density at voltage interval against the scan rates, a linear plot was obtained. The 
value of $1 / 2$ of the linear slope is $C_{\mathrm{dl}}$. EIS of the composites were evaluated by A.C. impedance at the frequency between $100 \mathrm{kHz}$ and $0.1 \mathrm{~Hz}$ with an amplitude of $5 \mathrm{mV}$ at overpotential of -300 and $300 \mathrm{mV}$ for HER and OER, respectively.

The TOF value is calculated by using the following formula:

$$
\begin{aligned}
& \mathrm{N}=\frac{Q}{2 F} \\
& \operatorname{TOF}\left(\mathrm{s}^{-1}\right)=\frac{I}{2 N F}
\end{aligned}
$$

Where $\mathrm{N}$ is the amounts of the active sites, $\mathrm{Q}$ is the electric quantity, and $\mathrm{I}$ is the catalytic current. $\mathrm{CV}$ plot was recorded in a phosphate buffer solution at $\mathrm{pH}=7.4$ from $-0.2 \mathrm{~V}$ to $0.6 \mathrm{~V} v s$. RHE with a scan rate of $50 \mathrm{mV} \mathrm{s}^{-1}$. 

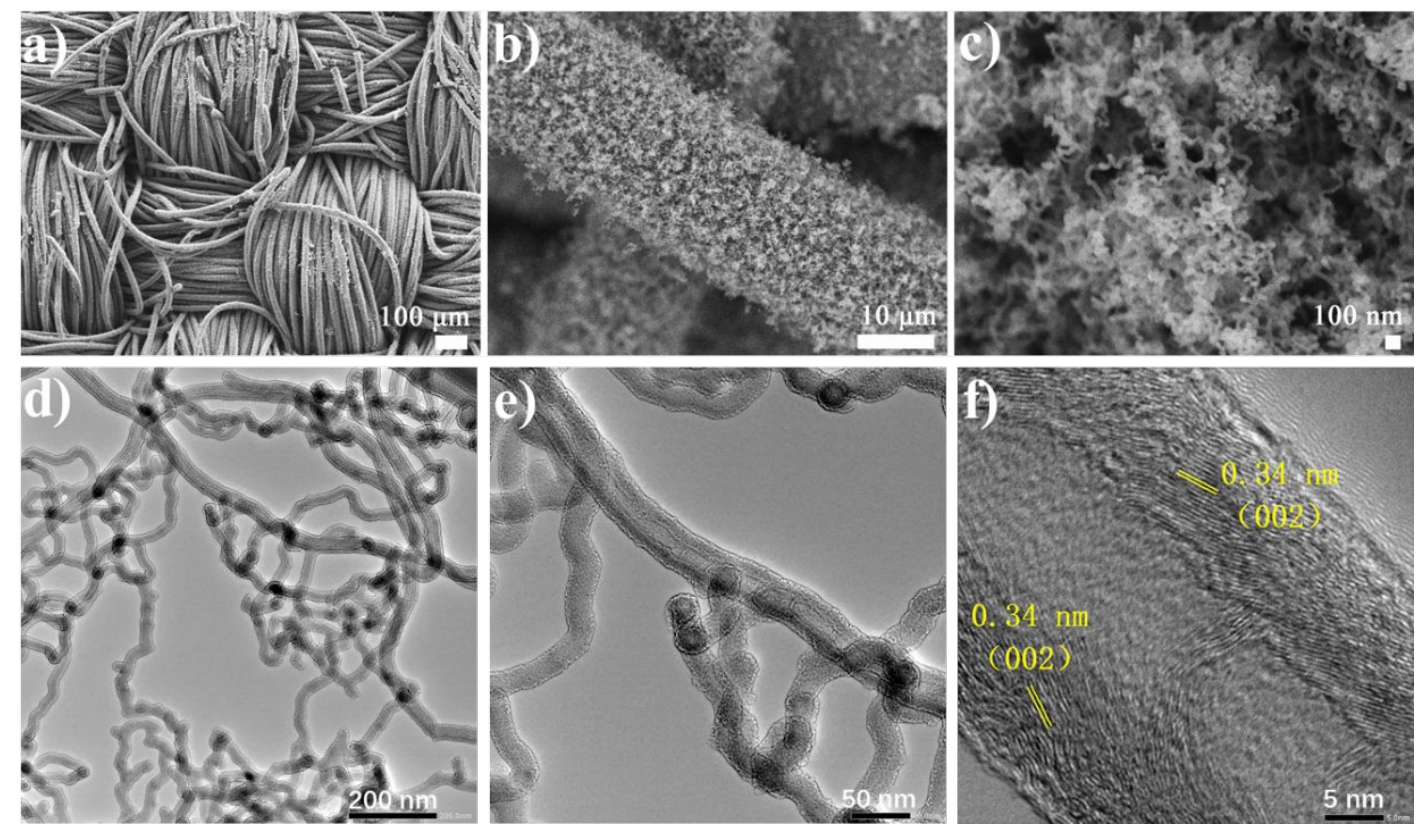

Figure S1. The (a-c) SEM images and (d-f) TEM images of the N-CNTs/CC

hierarchical substrate. 

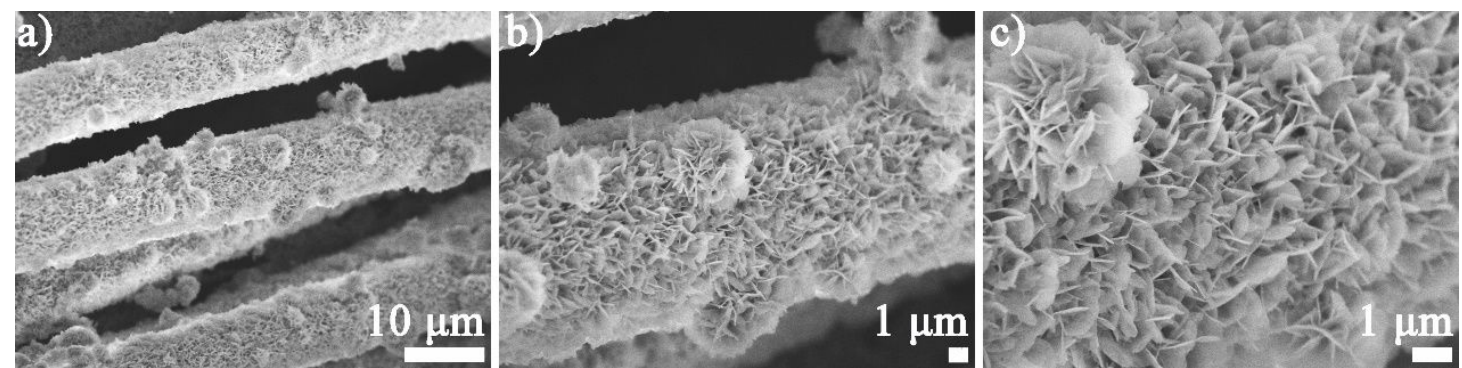

Figure S2. (a-c) SEM images of the MoNiFe/CC composite under different magnifications. 

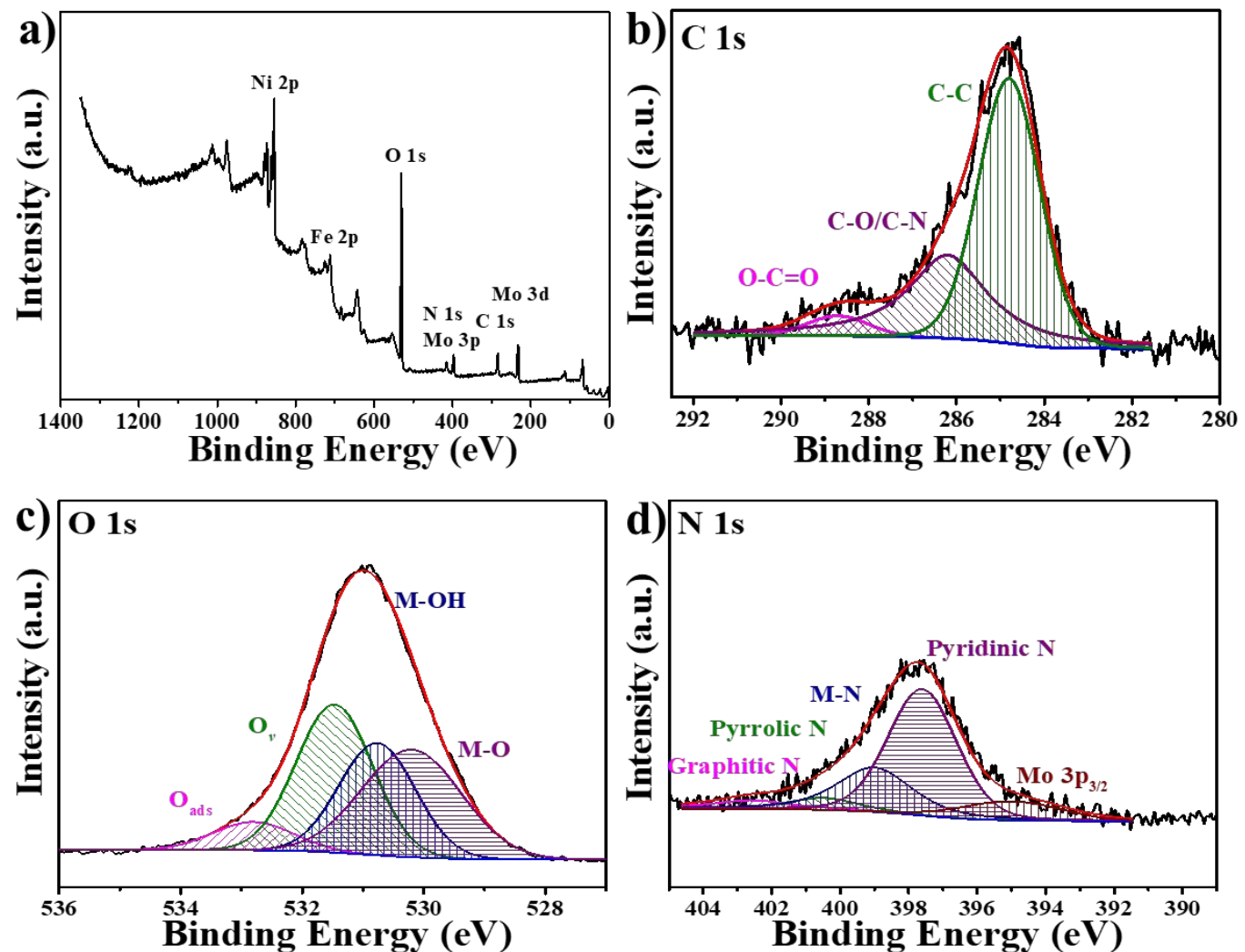

Figure S3. (a) Full XPS survey and the (b) C 1s, (c) O 1s and (d) N 1s spectra of the $\mathrm{MoNiFe} / \mathrm{N}-\mathrm{CNTs}$. 


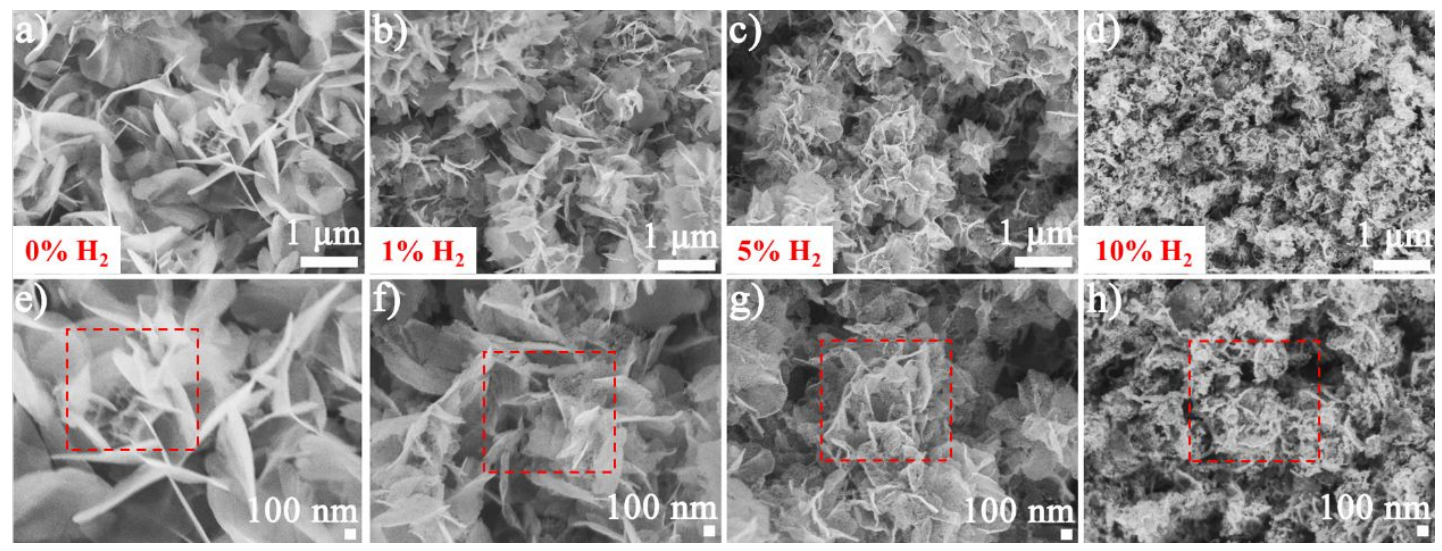

Figure S4. SEM images of the (a, e) H-MoNiFe/N-CNTs-0, (b, f) H-MoNiFe/NCNTs-1, (c, g) H-MoNiFe/N-CNTs-2 and (d, h) H-MoNiFe/N-CNTs-3. 

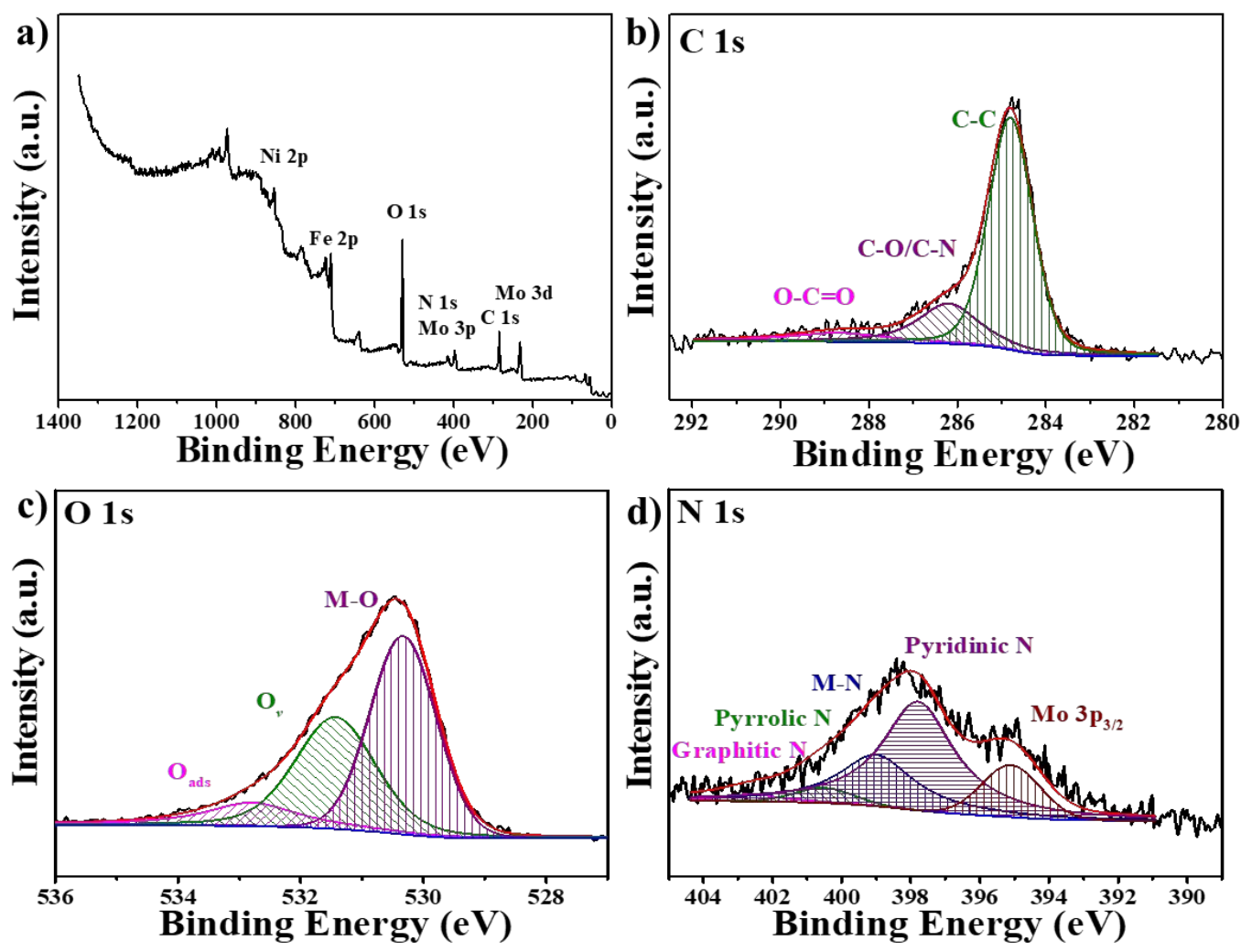

Figure S5. (a) Full XPS survey and the (b) C 1s, (c) O 1s and (d) N 1s spectra of the H-MoNiFe/N-CNTs-2. 


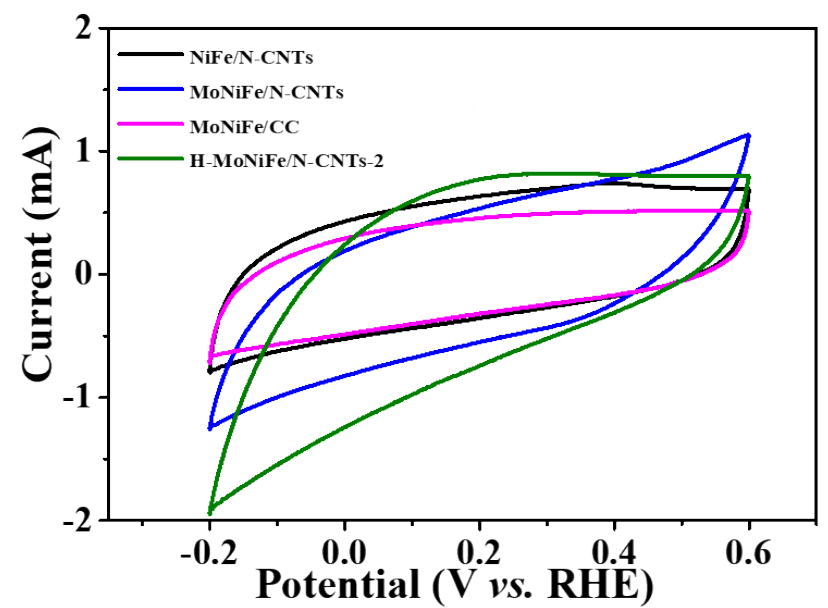

Figure S6. CVs curves recorded in a phosphate buffer solution at $\mathrm{pH}=7.4$ 

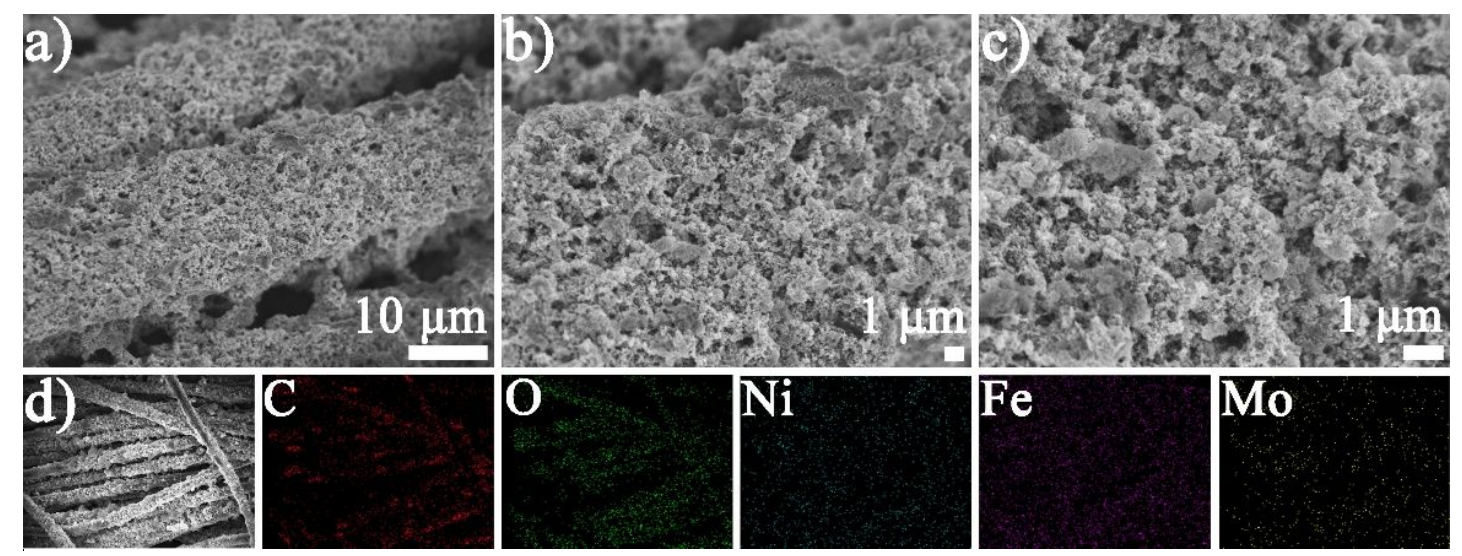

Figure S7. (a-c) SEM images under different magnifications, and (d) SEM image and related EDS mapping of the $\mathrm{H}-\mathrm{MoNiFe} / \mathrm{N}-\mathrm{CNTs}-2$ after long-term durability test. 

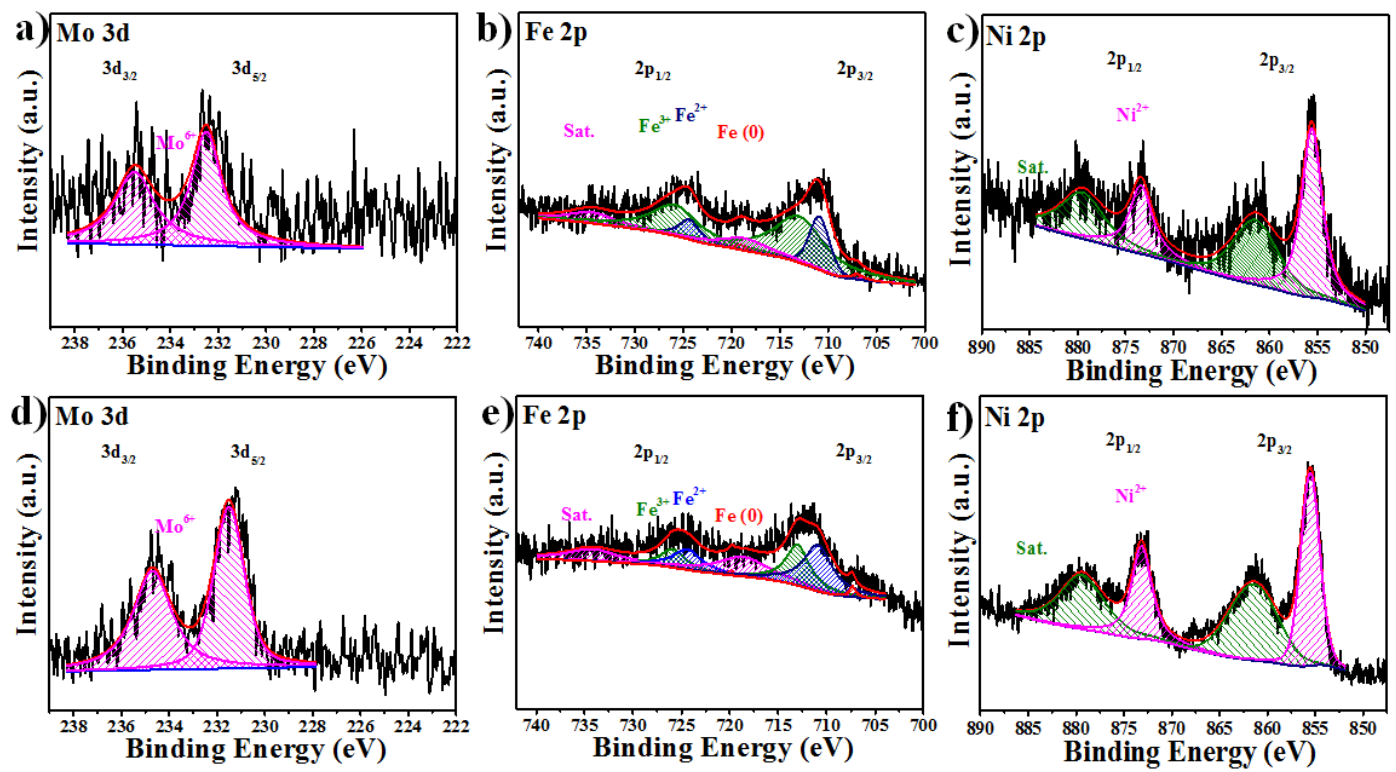

Figure S8. XPS spectrum of the (a-c) H-MoNiFe/N-CNTs-2 and (d-f) S-MoNiFe/NCNTs-1 after long-term durability test. 


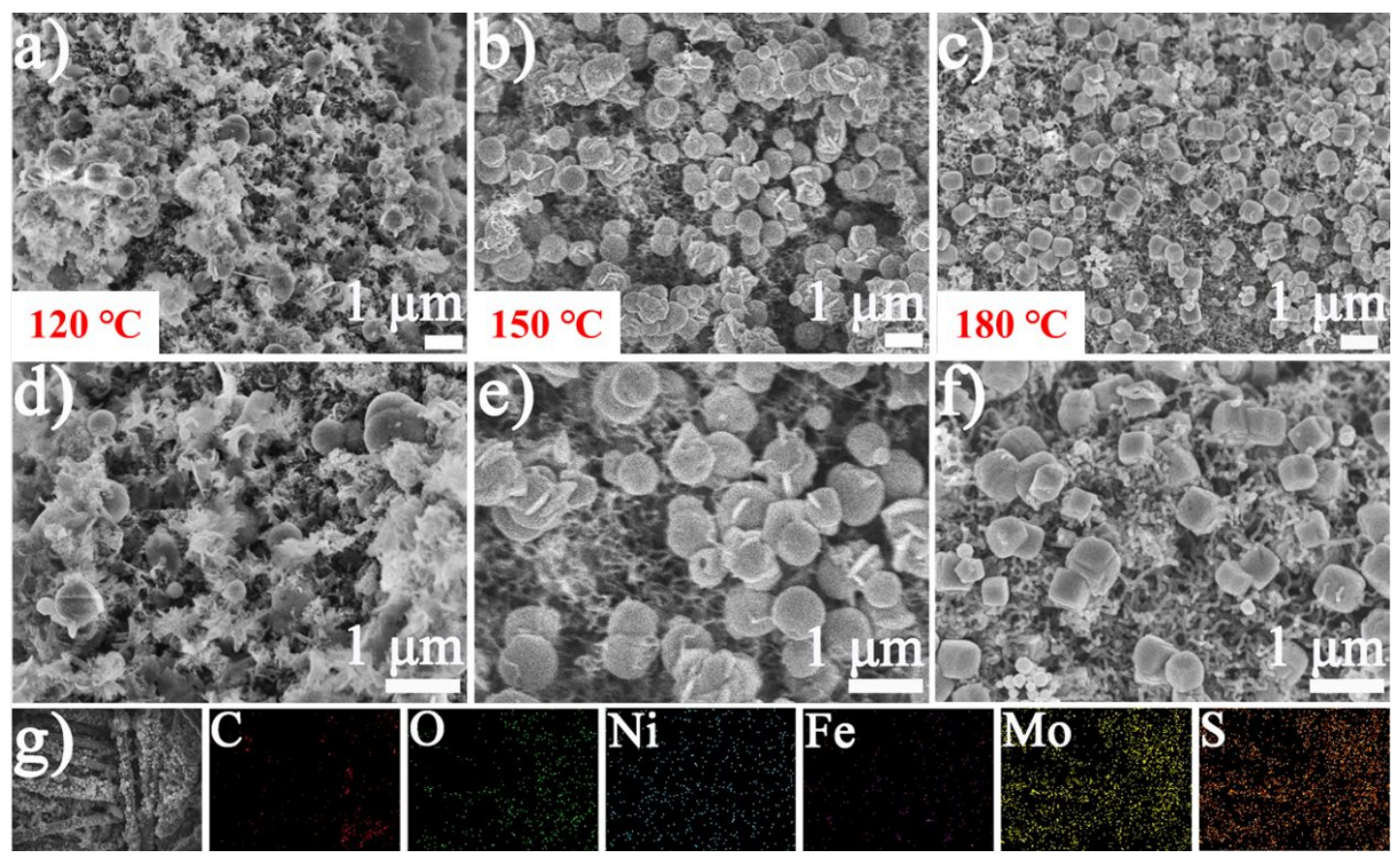

Figure S9. SEM images of (a, d) S-MoNiFe/N-CNTs-1, (b, e) S-MoNiFe/N-CNTs-2 and (c, f) S-MoNiFe/N-CNTs-3; g) EDS mapping of S-MoNiFe/N-CNTs-1. 

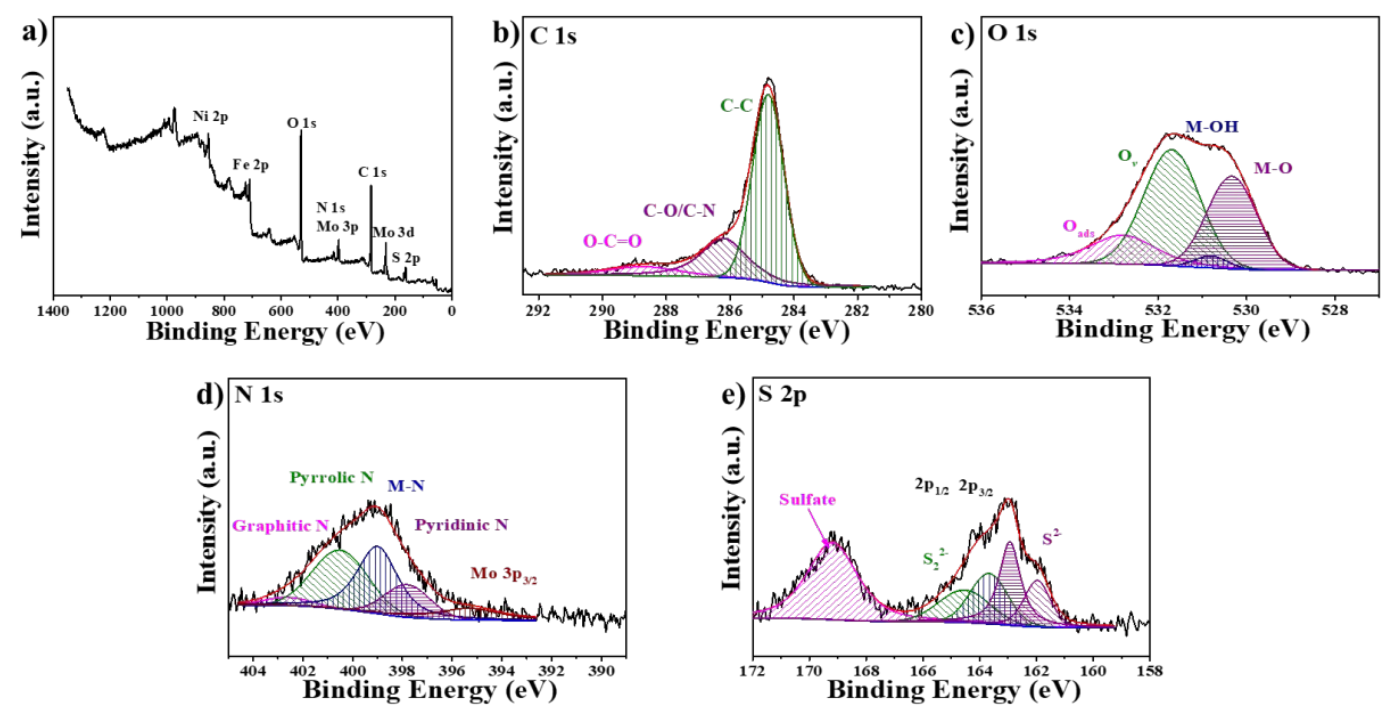

Figure S10. (a) Full XPS survey and the (b) C 1s, (c) O 1s, (d) N 1s and (e) S 2p spectra of the S-MoNiFe/N-CNTs-1. 

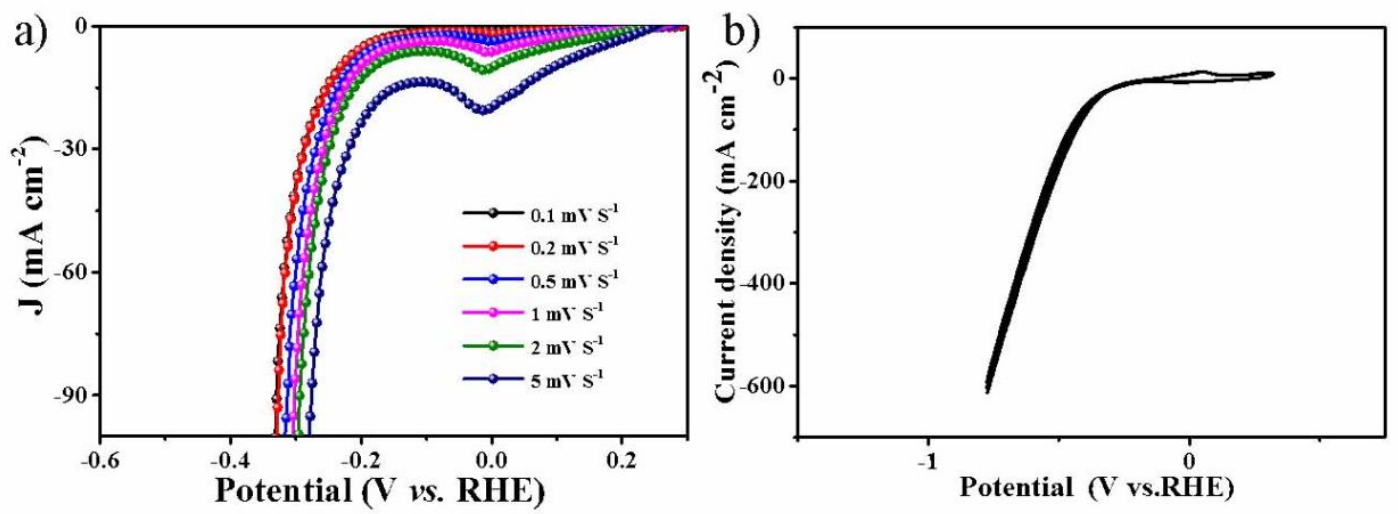

Figure S11. a) LSV curves at different scan rates; b) CV curve at $5 \mathrm{mV} / \mathrm{s}$ of the SMoNiFe/N-CNTs-1. 


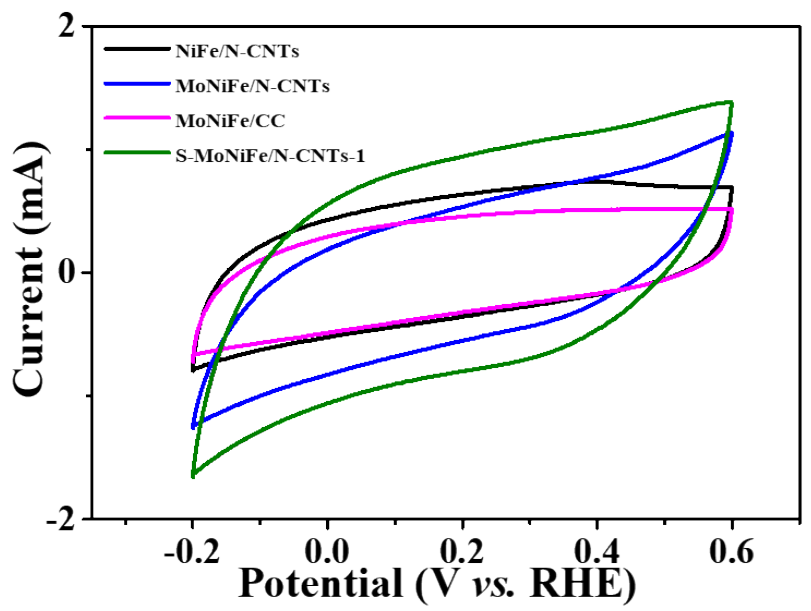

Fig. S12. $\mathrm{CV}$ s curves recorded in a phosphate buffer solution at $\mathrm{pH}=7.4$. 

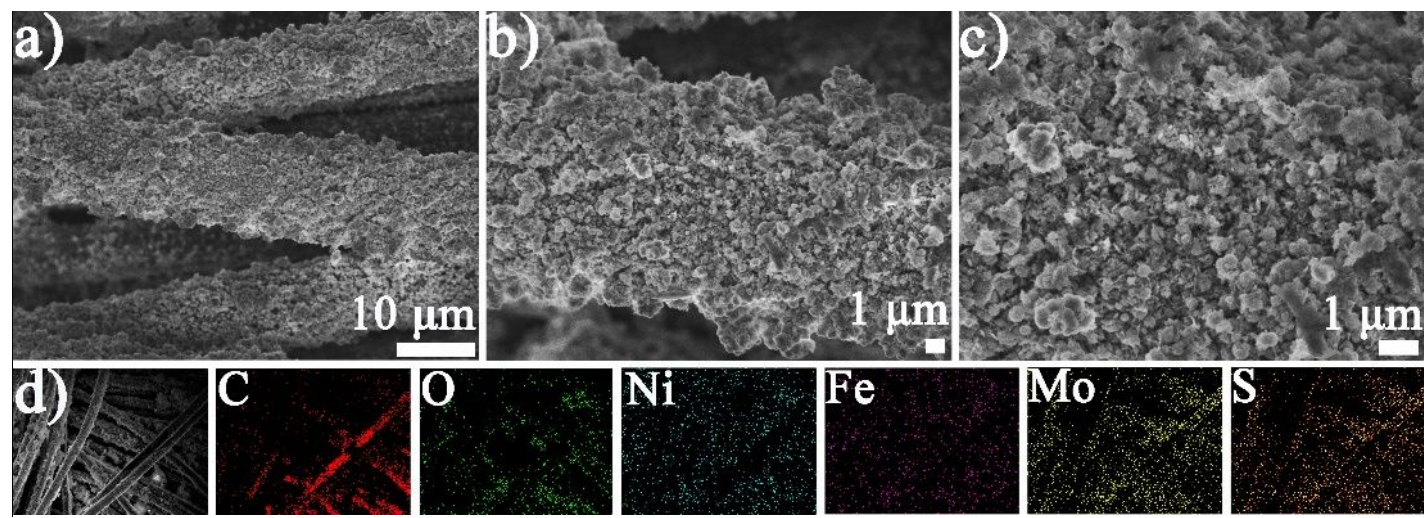

Figure S13. (a-c) SEM images under different magnifications, (d) SEM image and related EDS mapping of the S-MoNiFe/N-CNTs-1 after durability test. 
Table S1. Electrocatalytic OER performance of the catalysts in this work.

\begin{tabular}{|c|c|c|c|c|c|}
\hline Catalysts & $\begin{array}{c}J \\
(\mathrm{~mA} \mathrm{~cm}- \\
2)\end{array}$ & $\begin{array}{l}\text { Overpotential } \\
\qquad(\mathrm{mV})\end{array}$ & 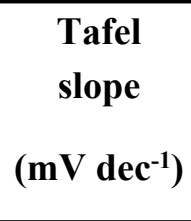 & 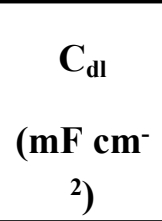 & $\begin{array}{l}\mathbf{R}_{\mathrm{ct}} \\
(\mathbf{\Omega})\end{array}$ \\
\hline $\mathrm{NiFe} / \mathrm{N}-\mathrm{CNTs}$ & 50 & 300.1 & 110.39 & 51.9 & 3.76 \\
\hline MoNiFe/N-CNTs & 50 & 281.5 & 61.0 & 67.5 & 2.09 \\
\hline $\mathrm{MoNiFe/CC}$ & 50 & 313.7 & 98.0 & 38.9 & 3.81 \\
\hline $\begin{array}{l}\text { H-MoNiFe/N-CNTs- } \\
0\end{array}$ & 50 & 306.1 & 82.2 & 49.7 & 2.97 \\
\hline $\begin{array}{l}\text { H-MoNiFe/N-CNTs- } \\
1\end{array}$ & 50 & 287.1 & 80.5 & 63.8 & 2.31 \\
\hline $\begin{array}{l}\text { H-MoNiFe/N-CNTs- } \\
2\end{array}$ & 50 & 259.0 & 44.3 & 76.9 & 1.13 \\
\hline $\begin{array}{l}\text { H-MoNiFe/N-CNTs- } \\
3\end{array}$ & 50 & 269.7 & 58.5 & 72.7 & 1.43 \\
\hline
\end{tabular}


Table S2. Electrocatalytic HER performance of the catalysts in this work.

\begin{tabular}{|c|c|c|c|c|c|}
\hline Catalysts & $\begin{array}{c}J \\
\left(\mathbf{m A ~ c m} \mathbf{c m}^{-}\right. \\
\left.{ }_{2}\right)\end{array}$ & $\begin{array}{c}\text { Overpotential } \\
(\mathrm{mV})\end{array}$ & 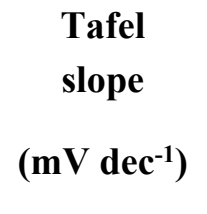 & $\begin{array}{c}\mathrm{C}_{\mathrm{dl}} \\
(\mathrm{mF} \mathrm{cm}- \\
\left.{ }_{2}\right)\end{array}$ & $\begin{array}{l}\mathbf{R}_{\mathrm{ct}} \\
(\boldsymbol{\Omega})\end{array}$ \\
\hline $\mathrm{NiFe} / \mathrm{N}-\mathrm{CNTs}$ & 10 & 310.5 & 162.3 & 51.9 & 29.75 \\
\hline MoNiFe/N-CNTs & 10 & 263.0 & 156.1 & 61.9 & 29.66 \\
\hline MoNiFe/CC & 10 & 353.4 & 174.9 & 38.9 & 49.64 \\
\hline $\begin{array}{l}\text { S-MoNiFe/N-CNTs- } \\
1\end{array}$ & 10 & 130.7 & 133.3 & 105.1 & 5.90 \\
\hline $\begin{array}{l}\text { S-MoNiFe/N-CNTs- } \\
2\end{array}$ & 10 & 189.9 & 157.8 & 100.3 & 17.89 \\
\hline $\begin{array}{l}\text { S-MoNiFe/N-CNTs- } \\
3\end{array}$ & 10 & 227.3 & 173.4 & 81.1 & 20.59 \\
\hline
\end{tabular}


Table S3. Comparison of the performance of reported trimetallic transition metal composites.

\begin{tabular}{|c|c|c|c|c|c|}
\hline Positive electrode//native electrode & electrolyte & $\begin{array}{c}\text { HER }(\eta) \\
\left(\mathbf{m V}\left(\mathbf{m A ~} \mathbf{c m}^{-2}\right)\right)\end{array}$ & 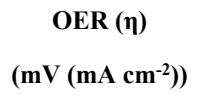 & $\begin{array}{c}\text { Overall }(\boldsymbol{\eta}) \\
\left(\mathbf{m V}\left(\mathbf{m A ~} \mathbf{c m}^{-2}\right)\right)\end{array}$ & ref \\
\hline NiFeMoS/NF-P//NiFeMoS/NF-P & $1 \mathrm{M} \mathrm{KOH}$ & $100(10)$ & $280(150)$ & $1.52(100)$ & 1 \\
\hline $\mathrm{Ni}_{2} \mathrm{Fe}_{1}-\mathrm{Mo} / / \mathrm{Ni}_{2} \mathrm{Fe}_{1}-\mathrm{Mo}$ & $1 \mathrm{M} \mathrm{KOH}$ & $84(10)$ & $231(10)$ & $1.62(10)$ & 2 \\
\hline $\begin{array}{l}\text { Mo-Ni } 2 \text { P@NiFe LDH/NF// } \\
\text { Mo-Ni }{ }_{2} \mathbf{P} @ N i F e ~ L D H / N F\end{array}$ & $1 \mathrm{M} \mathrm{KOH}$ & $82(10)$ & $269(40)$ & $1.46(10)$ & 3 \\
\hline $\begin{array}{l}\mathrm{Mo}-\mathrm{Ni}_{2} \mathrm{~S}_{3} @ \mathrm{NiFe} \mathrm{LDH} / / \\
\mathrm{Mo}-\mathrm{Ni}_{2} \mathrm{~S}_{3} @ \mathrm{NiFe} \text { LDH }\end{array}$ & $1 \mathrm{M} \mathrm{KOH}$ & $109(10)$ & $228(50)$ & $1.54(10)$ & 4 \\
\hline NiFe PVP//NiMo PVP & $1 \mathrm{M} \mathrm{KOH}$ & $130(10)$ & $297(10)$ & $1.66(10)$ & 5 \\
\hline $\mathrm{Fe}-\left(\mathrm{NiS}_{2} / \mathrm{MoS}_{2}\right) / \mathrm{Fe}-\left(\mathrm{NiS}_{2} / \mathrm{MoS}_{2}\right)$ & $1 \mathrm{M} \mathrm{KOH}$ & $87(10)$ & $234(10)$ & $1.51(10)$ & 6 \\
\hline NiFeMo//NiFeMo & $1 \mathrm{M} \mathrm{KOH}$ & $84.8(10)$ & $230(10)$ & $1.60(10)$ & 7 \\
\hline NP-FeCoNiMg-SS-900 & $1 \mathrm{M} \mathrm{KOH}$ & / & $254(10)$ & l & 8 \\
\hline SSF@NiFe-120//SSF@NiFe-120 & $1 \mathrm{M} \mathrm{KOH}$ & $100(10)$ & $210(10)$ & $1.55(10)$ & 9 \\
\hline $\begin{array}{l}\text { H-MoNiFe/N-CNTs-2// } \\
\text { S-MoNiFe/N-CNTs-1 }\end{array}$ & $1 \mathrm{M} \mathrm{KOH}$ & $130.7(10)$ & $259(50)$ & $1.58(10)$ & $\begin{array}{l}\text { This } \\
\text { work }\end{array}$ \\
\hline
\end{tabular}

\section{References}

1. Yan, K.-L.; Qin, J.-F.; Liu, Z.-Z.; Dong, B.; Chi, J.-Q.; Gao, W.-K.; Lin, J.-H.; Chai, Y.-M.; Liu, C.-G., Organic-inorganic hybrids-directed ternary NiFeMoS anemone-like nanorods with scaly surface supported on nickel foam for efficient overall water splitting. Chem. Eng. J. 2018, 334, 922-931.

2. Chen, Y.; Dong, C.; Zhang, J.; Zhang, C.; Zhang, Z., Hierarchically porous Mo-doped Ni-Fe oxide nanowires efficiently catalyzing oxygen/hydrogen evolution reactions. J. Mater. Chem. A 2018, 6 (18), 8430-8440.

3. Yang, Z.; Lin, Y.; Jiao, F.; Li, J.; Wang, J.; Gong, Y., In situ growth of 3D walnut-like nano-architecture Mo-Ni2P@NiFe LDH/NF arrays for synergistically enhanced overall water splitting. J. Energy Chem. 2020, 49, 189-197.

4. Feng, X.; Shi, Y.; Shi, J.; Hao, L.; Hu, Z., Superhydrophilic 3D peony flower-like Mo-doped Ni2S3@NiFe LDH heterostructure electrocatalyst for accelerating water splitting. Int. J. Hydrogen Energy 2021, 46 (7), 5169-5180.

5. Zhang, Y.; Xia, X.; Cao, X.; Zhang, B.; Tiep, N. H.; He, H.; Chen, S.; Huang, Y.; Fan, H. J., Ultrafine Metal Nanoparticles/N-Doped Porous Carbon 
Hybrids Coated on Carbon Fibers as Flexible and Binder-Free Water Splitting Catalysts. Adv. Energy Mater. 2017, 7 (15), 1700220.

6. Li, C.; Liu, M.; Ding, H.; He, L.; Wang, E.; Wang, B.; Fan, S.; Liu, K., A lightly Fe-doped (NiS2/MoS2)/carbon nanotube hybrid electrocatalyst film with laser-drilled micropores for stabilized overall water splitting and $\mathrm{pH}$-universal hydrogen evolution reaction. J. Mater. Chem. A 2020, 8 (34), 17527-17536.

7. Lv, Z.; Li, Z.; Tan, X.; Li, Z.; Wang, R.; Wen, M.; Liu, X.; Wang, G.; Xie, G.; Jiang, L., One-step electrodeposited NiFeMo hybrid film for efficient hydrogen production via urea electrolysis and water splitting. Appl. Surf. Sci. 2021, 552,149514 .

8. Hao, B.; Ye, Z.; Xu, J.; Li, L.; Huang, J.; Peng, X.; Li, D.; Jin, Z.; Ma, G., A high-performance oxygen evolution electrode of nanoporous Ni-based solid solution by simulating natural meteorites. Chem. Eng. J. 2021, 410, 128340.

9. Zhu, S.; Duan, G.; Chang, C.; Chen, Y.; Sun, Y.; Tang, Y.; Wan, P.; Pan, J., Fast Electrodeposited Nickle-Iron Hydroxide Nanosheets on Sintered Stainless Steel Felt as Bifunctional Electrocatalyts for Overall Water Splitting. ACS Sustain. Chem. Eng. 2020, 8 (26), 9885-9895. 